\title{
Osteoma osteoide de falange. Reporte de cinco casos y revisión bibliográfica
}

\author{
Santiago Argüelles, ${ }^{* \#}$ Fernando G. Ferrari, ${ }^{\star}$ Federico Reinares," Enrique Pereira," Carlos R. Zaidenberg ${ }^{*}$ \\ "Servicio de Ortopedia y Traumatología, Hospital Municipal de Vicente López "Bernardo Houssay", Buenos Aires, Argentina \\ ${ }^{* *}$ Servicio de Traumatología, Sanatorio Santa Fe, Santa Fe, Argentina \\ "Instituto Argentino de Diagnóstico y Tratamiento, Ciudad Autónoma de Buenos Aires, Argentina \\ ${ }^{*}$ Unidad de Estudio del Aparato Locomotor, $1^{a}$ Cátedra de Anatomía, Facultad de Medicina, Universidad Nacional de Buenos Aires, \\ Ciudad Autónoma de Buenos Aires, Argentina
}

\section{RESUMEN}

Introducción: El osteoma osteoide de falange es un cuadro infrecuente. El objetivo de este artículo es presentar cinco pacientes con diagnóstico histopatológico de osteoma osteoide de falange y resaltar los aspectos más relevantes del diagnóstico y el tratamiento. Materiales y Métodos: Estudio descriptivo, retrospectivo. Se evaluaron el dolor, según la escala analógica visual, la movilidad activa y el puntaje QuickDASH antes de la cirugía y en el control final a los 35.4 meses. Resultados: El tiempo medio desde la primera consulta hasta el diagnóstico fue de 10.6 meses (rango 5-16). El puntaje QuickDASH medio preoperatorio fue de 22,72 (rango 6,8-40,9). Luego del seguimiento medio de 35.4 meses (rango 17-63), todos los pacientes estaban satisfechos con el resultado. El puntaje medio de dolor fue 0. El puntaje QuickDASH medio fue 0. Conclusiones: El osteoma osteoide debe pensarse como diagnóstico diferencial ante un paciente que consulta por dolor digital subagudo o crónico que cede con antinflamatorios no esteroides. El tratamiento quirúrgico mediante el curetaje simple o la resección en bloque logra resultados satisfactorios. Sin embargo, como su prevalencia es baja, se suele pasar por alto y así se retrasan el diagnóstico y el tratamiento.

Palabras clave: Osteoma osteoide; falange; dedo; dolor crónico; tumefacción.

Nivel de Evidencia: IV

\section{Phalanx osteoid osteoma. Five-case report and literature review}

\section{ABSTRACT}

Introduction: Osteoid osteomas are rarely found in the phalanges. The purpose of this study is to report 5 cases with histologic diagnosis of phalanx osteoid osteoma and highlight the most important aspects of its diagnosis and treatment. Materials and Methods: A descriptive, retrospective study was conducted. Evaluation included pain level using the visual analog scale (VAS), active range of motion, and QuickDASH score before surgery and at final follow-up (mean, 35.4 months). Results: The mean time from first consultation to diagnosis was 10.6 months (range, 5-16 months). The mean preoperative QuickDASH score was 22.72 (range, 6.8-40.9). At last follow-up (mean, 35.4 months; range, 17-63), all patients were satisfied with the outcome. The mean VAS score for pain was 0 . The mean QuickDASH score was 0 . Conclusions: Osteoid osteoma should be considered in the differential diagnosis when patients complain of subacute or chronic digital pain that improves with NSAIDs. Surgical treatment by simple curettage or en bloc resection shows satisfactory results. However, due to its low prevalence, osteoid osteoma is commonly underdiagnosed resulting in diagnosis and treatment delays.

Key words: Osteoid osteoma; phalanx; finger; chronic pain; swelling.

Level of Evidence: IV

Recibido el 3-3-2020. Aceptado luego de la evaluación el 22-4-2020 • Dr. SANTIAGO ARGÜELLES • santiagoarguelles@yahoo.com (ID https://orcid.org/0000-0002-9002-5687 Cómo citar este artículo: Argüelles S, Ferrari FG, Reinares F, Pereira E, Zaidenberg CR. Osteoma osteoide de falange. Reporte de cinco casos y revisión bibliográfica. Rev Asoc Argent Ortop Traumatol 2020;85(4):335-348. https://doi.org/10.15417/issn.1852-7434.2020.85.4.1064 


\section{INTRODUCCIÓN}

El osteoma osteoide (OO) fue descrito por Jaffe, en $1935 .{ }^{1}$ Se trata de un tumor benigno, osteoblástico, cuya característica histológica es un centro nuclear de compuesto osteoide con grado variable de calcificación (nido), delimitado por un halo óseo reactivo. ${ }^{1,2}$ Representa el $10 \%$ de los tumores óseos benignos. ${ }^{3}$ Se manifiesta habitualmente en niños y adultos jóvenes, con una incidencia mayor en hombres que en mujeres (relación 2:1). La manifestación en la mano representa el 8\% del total, y los metacarpianos son los huesos más afectados. ${ }^{4}$ A pesar de su pequeño tamaño, el OO puede provocar síntomas intensos, probablemente debido a la secreción de prostaglandinas por parte de las células tumorales ${ }^{5,6} \mathrm{El}$ compromiso de las falanges es infrecuente, ${ }^{7-9}$ se registran casos aislados que, debido a su inusual localización, manifestación clínica y radiológica, suelen retrasar el diagnóstico y el tratamiento., $2,-11$

El objetivo de este estudio es presentar cinco pacientes con diagnóstico histopatológico de $\mathrm{OO}$ de falange y resaltar los aspectos más importantes en el diagnóstico y el tratamiento de la enfermedad.

\section{MATERIALES Y MÉTODOS}

Se realizó un estudio descriptivo retrospectivo. Se presentan cinco pacientes con diagnóstico de OO de falange, confirmado por estudio anatomopatológico diferido, operados entre julio de 2015 y febrero de 2018. Las lesiones se localizaron mediante radioscopia intraoperatoria antes de la resección (Figura 1).

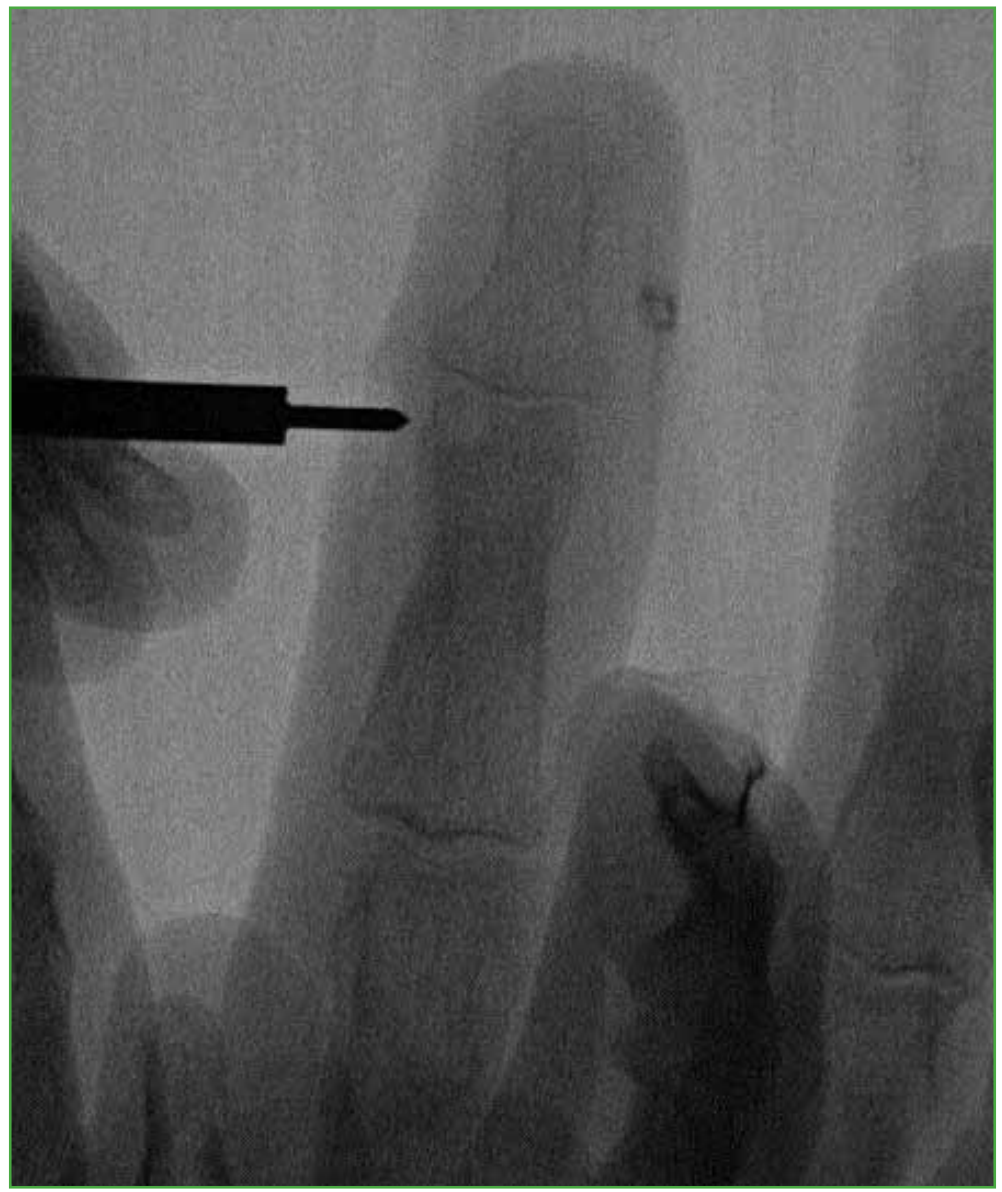

Figura 1. Caso 2. Localización intraoperatoria de la lesión mediante radioscopia. 
En tres pacientes, se efectuó un abordaje lateral (casos 1, 2 y 5) y, en dos, un abordaje volar de tipo Brunner (casos 3 y 4). El caso 4 fue operado inicialmente mediante un abordaje dorsal y se realizó un curetaje simple de la lesión. A los dos meses y ante los síntomas persistentes, la falta de un diagnóstico anatomopatológico de certeza y la confirmación por tomografía computarizada (TC) de la persistencia del nido, se procedió a la resección en bloque de la lesión mediante un abordaje volar de tipo Brunner. Un paciente fue sometido a un curetaje simple de la lesión (caso 1); otro, a curetaje y relleno con injerto autólogo de radio distal (caso 2); dos, a resección en bloque más injerto con sustituto óseo (casos 3 y 5) y otro, a resección en bloque sin la colocación de injerto (caso 4). Los pacientes fueron inmovilizados con férula digital durante tres semanas, seguida de terapia física. El seguimiento medio fue de 35.4 meses (rango 17- 63). Antes de la cirugía, se registraron el tiempo transcurrido entre la primera consulta y el diagnóstico, los síntomas y signos de presentación, el dolor según la escala analógica visual (EAV), en la cual 0 indica sin dolor y 10, el peor dolor. Se utilizó también el puntaje del cuestionario QuickDASH, donde 0 indica el mejor puntaje y 100, el peor. Los pacientes fueron citados para la evaluación clínica y radiográfica final en septiembre de 2019. Se registraron la movilidad activa mediante goniometría, el dolor según la EAV y se completó el cuestionario QuickDASH.

\section{RESULTADOS}

Cuatro de los cinco pacientes eran varones. Tres tenían compromiso de la falange proximal con localización intracortical; uno, de la falange media y uno, de la falange distal, ambos de localización esponjosa (Figura 2).

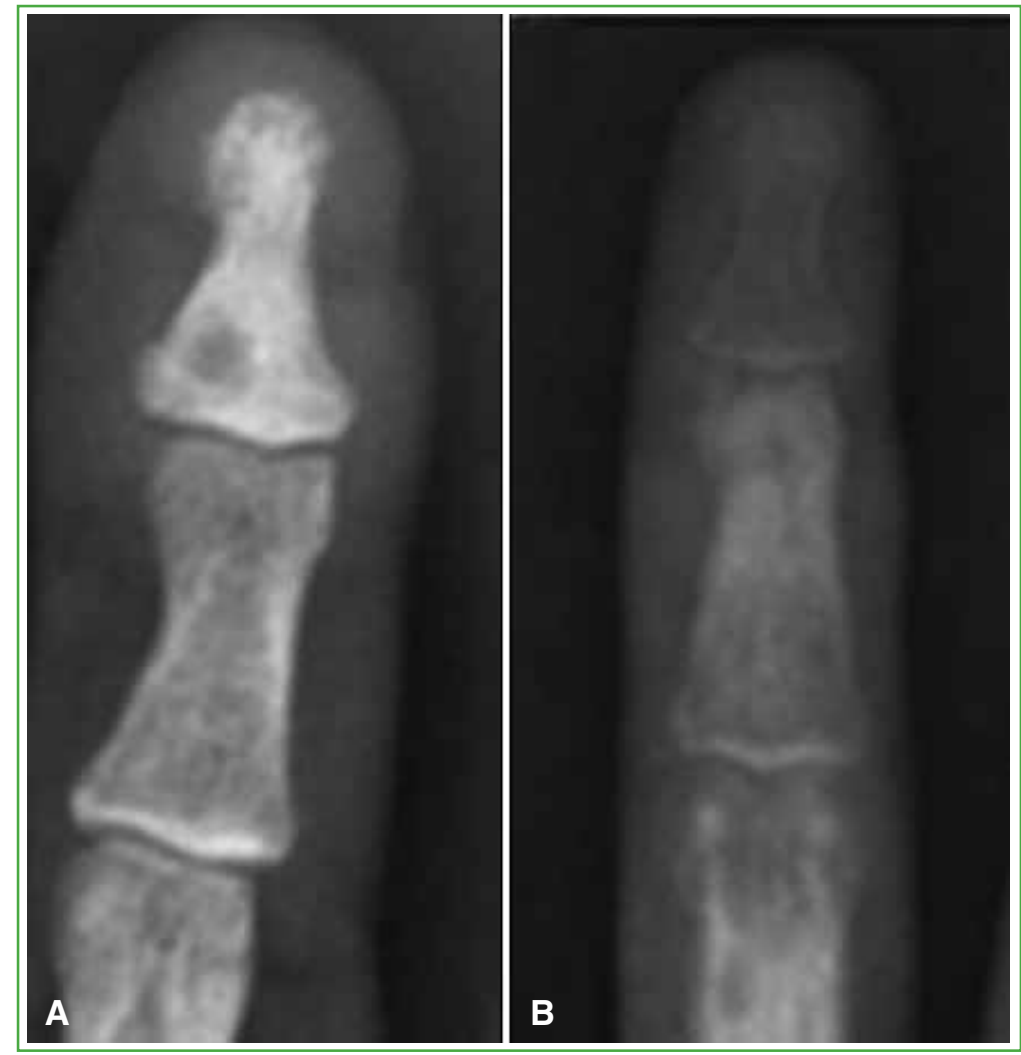

Figura 2. A. Caso 1. Osteoma osteoide de localización esponjosa. Radiografía de dedo meñique derecho. Imagen osteolítica en la base de la tercera falange. No se observa un halo esclerótico ni calcificación central. B. Caso 2. Imagen osteolítica con pequeña calcificación central en el cóndilo radial de la segunda falange. 
Un paciente fue sometido a un curetaje simple de la lesión (Figura 3); otro, a curetaje y relleno con injerto óseo; a dos se les efectuó una resección en bloque con injerto óseo (Figura 4) y a otro, una resección en bloque sin colocación de injerto.

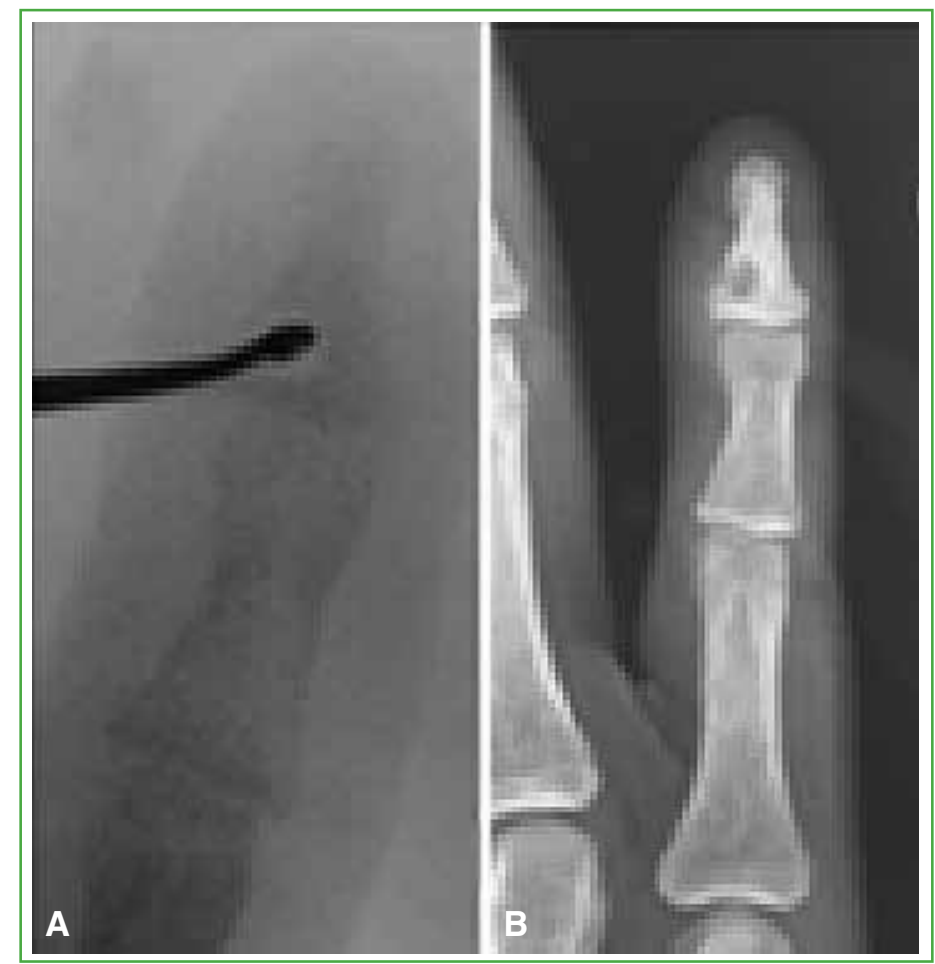

Figura 3. A. Caso 1. Curetaje de la lesión mediante un abordaje lateral.

B. Radiografía posoperatoria.
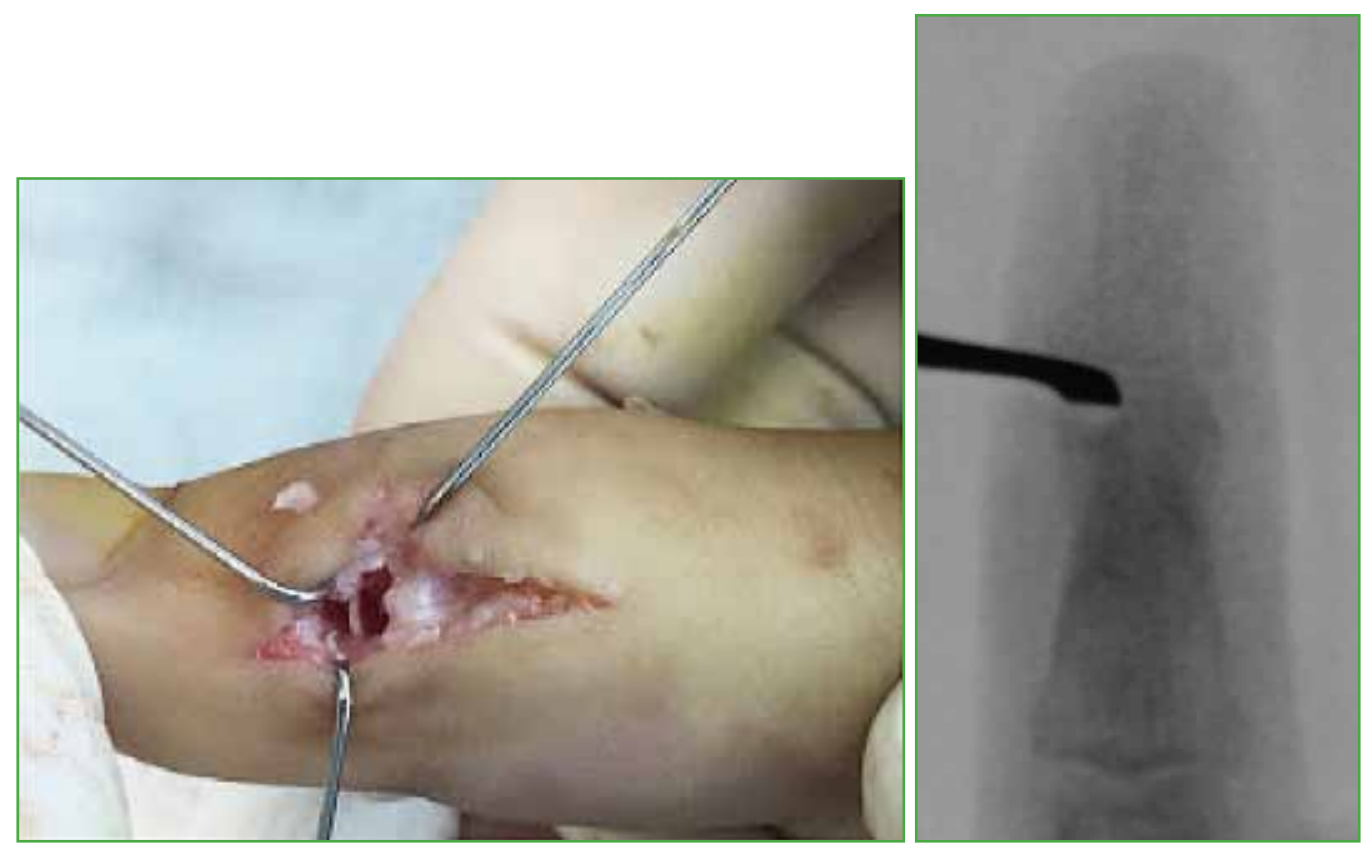

Figura 4. Caso 2. Curetaje de la lesión mediante un abordaje lateral. Se preservó el cartílago articular. El defecto fue llenado con injerto esponjoso de radio distal. 
Un paciente (caso 4) requirió un segundo procedimiento por resección incompleta. El tamaño medio de las lesiones en su diámetro máximo medidos por TC fue de 5,1 mm (rango 2,9-8,14) (Tabla).

Tabla. Datos de los pacientes y resultados

\begin{tabular}{|c|c|c|c|c|c|c|c|c|c|c|c|c|}
\hline Caso & Edad & Sexo & $\begin{array}{l}\text { Demora } \\
\text { diag- } \\
\text { nóstica } \\
\text { (meses) }\end{array}$ & Clínica & $\begin{array}{l}\text { Localiza- } \\
\text { ción }\end{array}$ & $\begin{array}{l}\text { Tamaño } \\
\text { (diámetro } \\
\text { máximo) }\end{array}$ & $\begin{array}{l}\text { Trata- } \\
\text { miento }\end{array}$ & $\begin{array}{l}\text { Dolor } \\
\text { preope- } \\
\text { ratorio } \\
\text { (EAV) }\end{array}$ & $\begin{array}{l}\text { Dolor } \\
\text { posope- } \\
\text { ratorio } \\
\text { (EAV) }\end{array}$ & $\begin{array}{l}\text { Puntaje } \\
\text { Quick- } \\
\text { DASH } \\
\text { preopera- } \\
\text { torio }\end{array}$ & $\begin{array}{l}\text { Puntaje } \\
\text { Quick- } \\
\text { DASH } \\
\text { posope- } \\
\text { ratorio }\end{array}$ & $\begin{array}{l}\text { Complica- } \\
\text { ciones }\end{array}$ \\
\hline 1 & 14 & M & 16 & $\begin{array}{l}\text { Dolor } \\
\text { nocturno, } \\
\text { dolor } \\
\text { palpatorio, } \\
\text { aumento del } \\
\text { diámetro, uña } \\
\text { en vidrio de } \\
\text { reloj }\end{array}$ & $\begin{array}{l}\text { Base } \\
\text { falange 3, } \\
\text { esponjosa }\end{array}$ & $6,1 \mathrm{~mm}$ & $\begin{array}{l}\begin{array}{l}\text { Abordaje } \\
\text { lateral }\end{array} \\
\begin{array}{l}\text { Curetaje } \\
\text { simple }\end{array}\end{array}$ & $6 / 10$ & 0 & 25 & 0 & No \\
\hline 2 & 24 & M & 14 & $\begin{array}{l}\text { Dolor } \\
\text { continuo, } \\
\text { dolor } \\
\text { palpatorio, } \\
\text { hiperhidrosis, } \\
\text { artritis } \\
\text { interfalángica } \\
\text { distal }\end{array}$ & $\begin{array}{l}\text { Cóndilo } \\
\text { falange 2, } \\
\text { esponjosa } \\
\text { subcondral }\end{array}$ & $3,3 \mathrm{~mm}$ & $\begin{array}{l}\text { Abordaje } \\
\text { lateral }\end{array}$ & $8 / 10$ & 0 & 22,7 & 0 & No \\
\hline 3 & 16 & F & 12 & $\begin{array}{l}\text { Dolor } \\
\text { continuo, } \\
\text { aumento del } \\
\text { diámetro, } \\
\text { tenosinovitis } \\
\text { de flexores }\end{array}$ & $\begin{array}{l}\text { Falange 1, } \\
\text { intracortical } \\
\text { volar }\end{array}$ & $2,9 \mathrm{~mm}$ & $\begin{array}{l}\text { Abordaje } \\
\text { volar } \\
\text { Resección } \\
\text { en bloque } \\
\text { más } \\
\text { relleno } \\
\text { con } \\
\text { sustituto } \\
\text { óseo }\end{array}$ & $6 / 10$ & 0 & 18,2 & 0 & No \\
\hline 4 & 13 & M & 5 & $\begin{array}{l}\text { Dolor, } \\
\text { aumento del } \\
\text { diámetro, } \\
\text { limitación de } \\
\text { la flexión }\end{array}$ & $\begin{array}{l}\text { Falange 1, } \\
\text { intracortical } \\
\text { volar }\end{array}$ & $8,14 \mathrm{~mm}$ & $\begin{array}{l}\begin{array}{l}\text { Abordaje } \\
\text { volar }\end{array} \\
\text { Resección } \\
\text { en bloque } \\
\text { (sin injerto } \\
\text { óseo) }\end{array}$ & $4 / 10$ & 0 & 40,9 & 0 & $\begin{array}{l}\text { Requirió } \\
\text { un segundo } \\
\text { procedi- } \\
\text { miento por } \\
\text { resección } \\
\text { incompleta }\end{array}$ \\
\hline 5 & 27 & M & 6 & $\begin{array}{l}\text { Dolor, dolor } \\
\text { palpatorio, } \\
\text { aumento del } \\
\text { diámetro }\end{array}$ & $\begin{array}{l}\text { Falange 1, } \\
\text { intracortical } \\
\text { medial }\end{array}$ & $\begin{array}{l}\text { No } \\
\text { evaluado }\end{array}$ & $\begin{array}{l}\begin{array}{l}\text { Abordaje } \\
\text { lateral }\end{array} \\
\text { Curetaje } \\
\text { y relleno } \\
\text { con } \\
\text { sustituto } \\
\text { óseo }\end{array}$ & 3 & 0 & 6,8 & 0 & No \\
\hline
\end{tabular}

$\mathrm{M}=$ masculino, $\mathrm{F}=$ femenino, $\mathrm{EAV}=$ escala analógica visual. 
La edad media de presentación fue de 18.8 años (rango 14- 27). El tiempo promedio transcurrido entre la primera consulta y el diagnóstico fue de 10.6 meses (rango 5-16). Todos consultaron por dolor con un puntaje medio de 5,4 (rango 3- 8) según la EAV. Todos tenían tumefacción digital (Figura 5). El puntaje QuickDASH medio preoperatorio era de 22,72 (rango 6,8-40,9). Tras un seguimiento medio de 35.4 meses (rango 17-63), ninguno tenía síntomas. Los puntajes de dolor según la EAV y el cuestionario QuickDASH fueron 0. La movilidad activa fue completa en todos los casos (Figuras 6 y 7). El caso 4 requirió un segundo procedimiento por resección incompleta (Tabla).

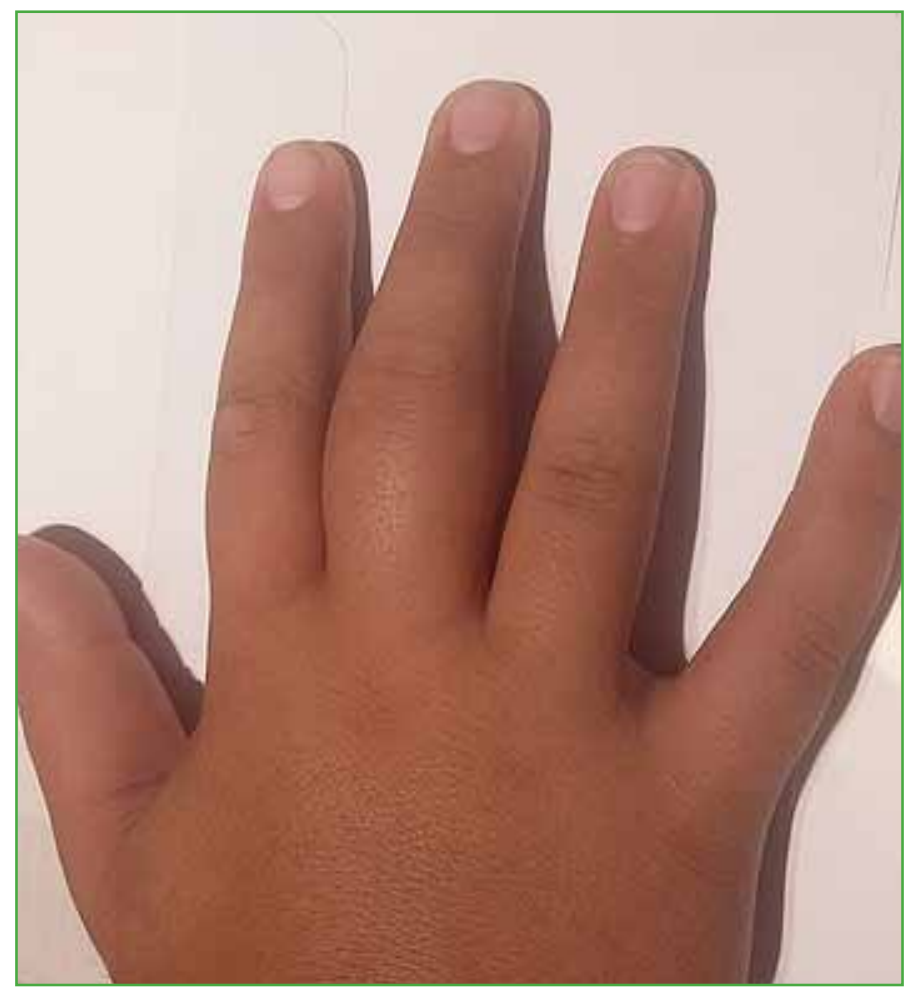

Figura 5. Caso 4. Nótese la tumefacción en el tercer dedo. Paciente con osteoma osteoide en la segunda falange, de localización intracortical.

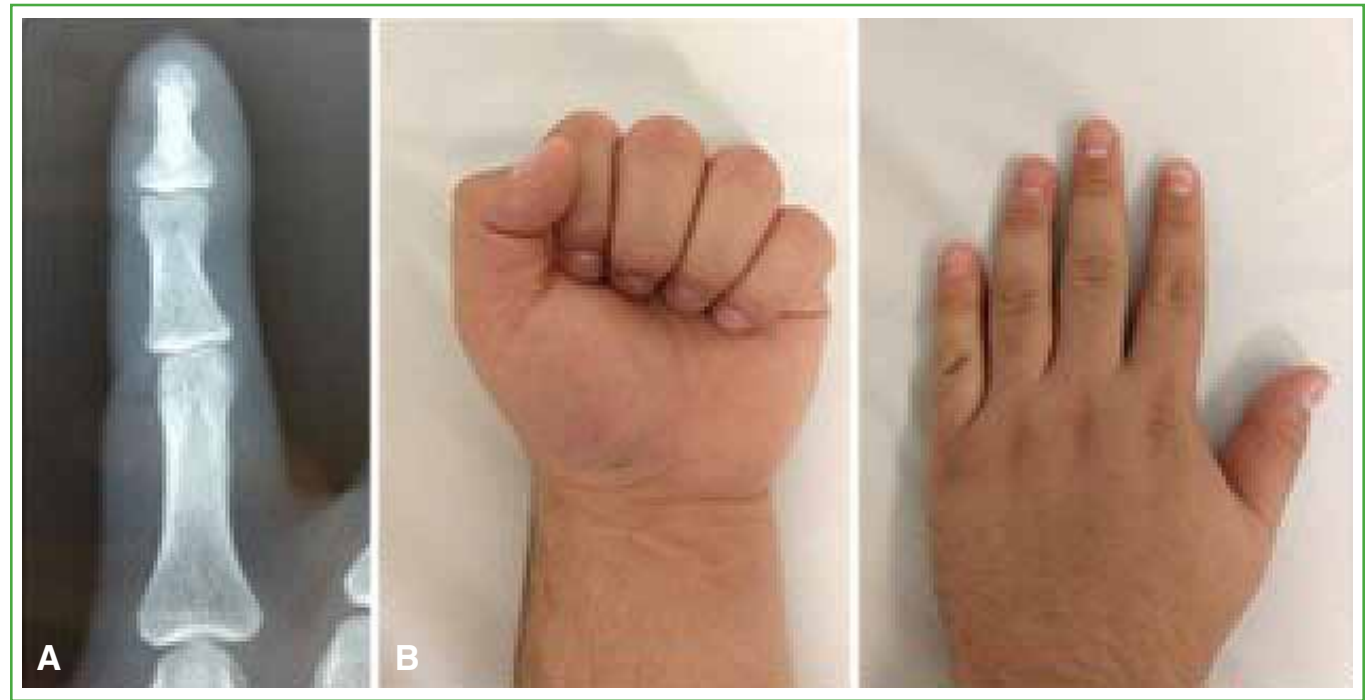

Figura 6. Caso 1. A. Remodelación ósea completa. B. Movilidad activa tras 17 meses de seguimiento. 


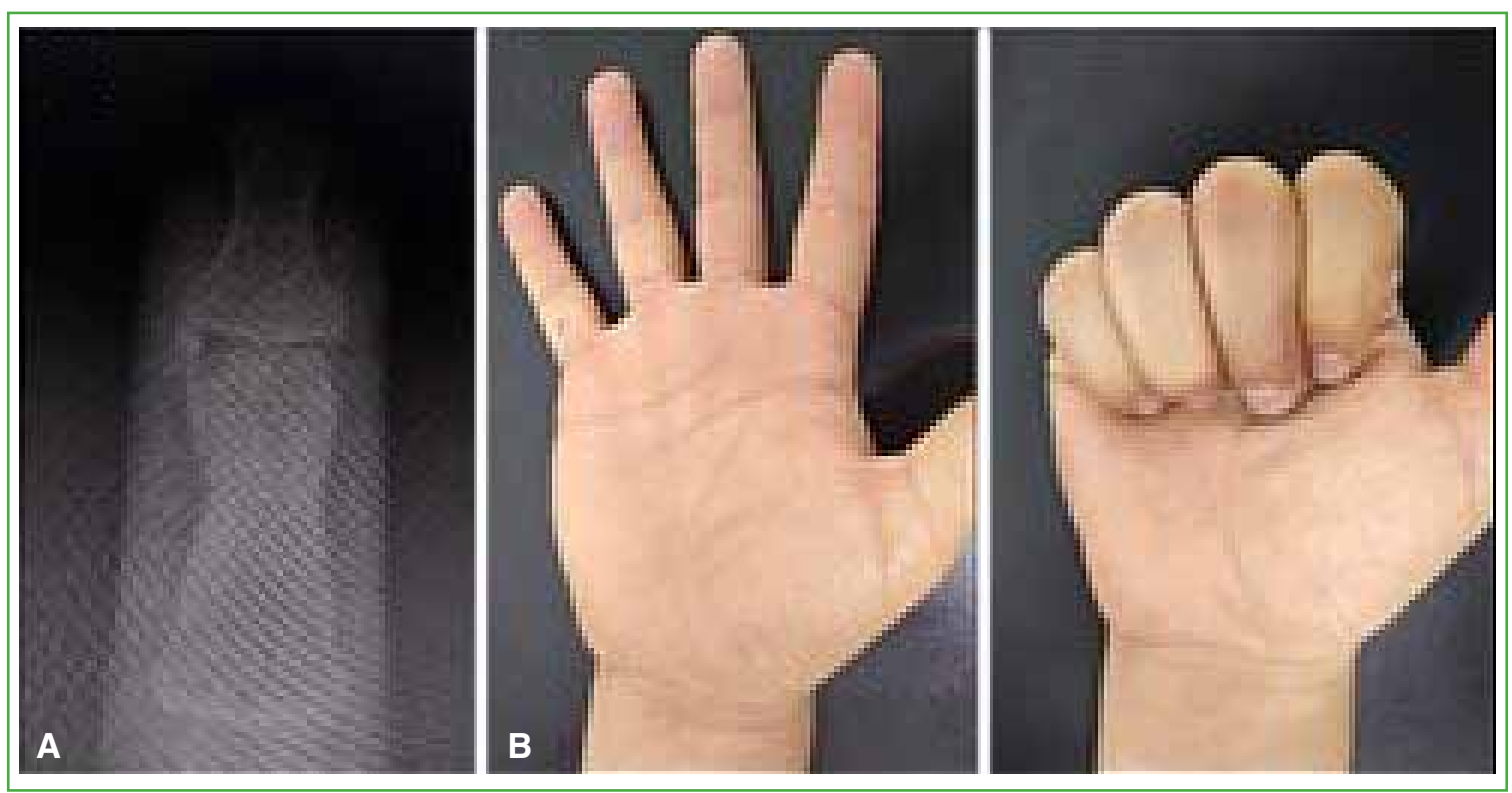

Figura 7. Caso 2 a los 20 meses de la cirugía. A. Resorción parcial del injerto. B. Movilidad final.

\section{DISCUSIÓN}

El OO de falange es un cuadro infrecuente. ${ }^{7,8}$ Clínicamente se caracteriza por un dolor progresivo de predominio nocturno que, en ocasiones, puede ser continuo. ${ }^{8,11}$ Habitualmente cede ante la administración de salicilatos y otros antinflamatorios no esteroides. . $^{2,-6,8,11}$

La etiología del dolor producido por el OO podría ser multifactorial. El nivel de prostaglandinas en el nido puede ser de 100 a 1000 veces más alto que el del tejido normal, lo cual induce la vasodilatación y el aumento de la permeabilidad capilar en los tejidos que rodean la lesión. ${ }^{11}$ Nakatsuchi y cols. han detectado un conjunto de fibras nerviosas en la lesión y en su periferia acompañando a los vasos sanguíneos o como fibras independientes, lo que sugiere que el dolor es mediado por el sistema nervioso a través de este haz de fibras. ${ }^{12}$ El dolor podría también reflejar los cambios en la presión de los vasos y deberse a la irritación directa de las fibras nerviosas incluidas cerca del nido. ${ }^{13}$ La hipertrofia de los tejidos blandos es motivo de controversia, puede ser causada por el metabolismo acelerado del tumor. ${ }^{14,15}$

Se han descrito tanto el cierre prematuro de la fisis como el aumento del crecimiento cuando la falange distal está afectada. ${ }^{11,15-17}$ Las manifestaciones clínicas del OO en falanges pueden ser tan atípicas que incluso puede ser indoloro. La sinovitis y la limitación de la movilidad son manifestaciones frecuentes cuando la localización es epifisaria yuxtarticular. ${ }^{7,11,18}$

El OO puede clasificarse según su ubicación en cortical, esponjoso y subperióstico. ${ }^{19}$ La imagen radiográfica típica cortical comprende un área radiolúcida $<1 \mathrm{~cm}$ de diámetro, de ubicación subcortical asociada con esclerosis ósea adyacente densa y reacción perióstica. En general, se detecta un nido centralmente dentro de la radiolucidez. En la variedad esponjosa, suele haber un área radiolúcida, pero con poca o ninguna esclerosis adyacente. La variedad menos común es la subperióstica que aparece como una imagen de entre 0,5 y $1 \mathrm{~cm}$ de diámetro inmediatamente adyacente al hueso. En estos casos, la cortical puede demostrar un defecto por presión o hueso irregular por resorción. ${ }^{19}$

Si bien la clasificación según su localización es válida en las falanges, es importante aclarar que el tamaño de las lesiones antes mencionadas corresponde a otras localizaciones en el esqueleto, su tamaño es significativamente menor en las falanges; en nuestra serie, fue de entre 2,9 y 8,1 mm medido por TC en su diámetro máximo.

La variedad cortical del $\mathrm{OO}$ es la más frecuente, pero la subperióstica y la esponjosa serían más frecuentes en los huesos pequeños de la mano y el pie., ${ }^{918}$

En las falanges, las imágenes radiográficas, con frecuencia, pueden ser atípicas y, en ocasiones, únicamente se observa osteólisis,,${ }^{11,15,16,20}$ e incluso pueden ser negativas $;{ }^{14}$ en estas situaciones, la TC, la resonancia magnética (RM) y, a veces, la gammagrafía ${ }^{9,18}$ juegan un papel significativo para el diagnóstico presuntivo. ${ }^{5,6}$ 
La TC es útil cuando no se logra detectar el nido en las radiografías simples, tiene la mejor especificidad para identificar y caracterizar la lesión (Figuras 8 y 9). ${ }^{21}$ Sugerimos solicitar una TC helicoidal de alta resolución con cortes del menor espesor posible (habitualmente $1 \mathrm{~mm}$ ) en las falanges, ya que, por su pequeño tamaño, el OO podría pasar inadvertido.

Los hallazgos tomográficos típicos son los siguientes:

- una zona de baja densidad redonda u ovalada correspondiente al nido,

- un área de alta densidad dentro del nido, de mayor o menor tamaño según su grado de calcificación,

- un halo de esclerosis periférica reactiva o reacción perióstica (poco frecuente en las falanges).

La RM es un estudio más sensible que la TC para detectar el OO en las falanges; ;1,22 además, puede proporcionar detalles adicionales en relación con la reacción inflamatoria de los tejidos blandos adyacentes a la lesión. Cuanto más cerca esté la lesión de la zona medular, mayor será el papel de la RM en el reconocimiento del nido en comparación con la TC. Las señales en la RM son diferentes entre la médula ósea, el nido, la esclerosis ósea reactiva y los tejidos blandos. El nido aparece en la secuencia T1 como un área de señal hipo o isointensa, con intensidad variable en T2, según el grado de mineralización. Si hay esclerosis reactiva periférica, se observa como una señal hipointensa en T1 y en T2. Típicamente se puede visualizar edema en la médula ósea y en los tejidos blandos que rodean la lesión (Figura 10). ${ }^{23,24}$ Cuando el nido está parcialmente mineralizado, se puede ver una imagen en escarapela. La administración de gadolinio aumenta notablemente la visibilidad del nido debido a su alta vascularización..$^{22}$

Cuando no se observa hueso reactivo periférico, el nido puede suele ser difícil de detectar. En estas situaciones, la gammagrafía ósea adquiere utilidad diagnóstica, tiene una sensibilidad cercana al 100\% para detectar estas lesiones, y muestra un aumento de la captación en las tres fases (Figura 11). ${ }^{25}$

El OO tiene un gran suministro vascular y un número superior al normal de osteoblastos; por lo tanto, el tumor toma ávidamente el tecnecio 99 inyectado que se absorbe sobre los cristales de hidroxiapatita. El signo de la doble densidad (también conocido como el punto más cálido dentro del área caliente) es diagnóstico de $\mathrm{OO}$ y es muy útil para distinguirlo de la osteomielitis. ${ }^{26}$

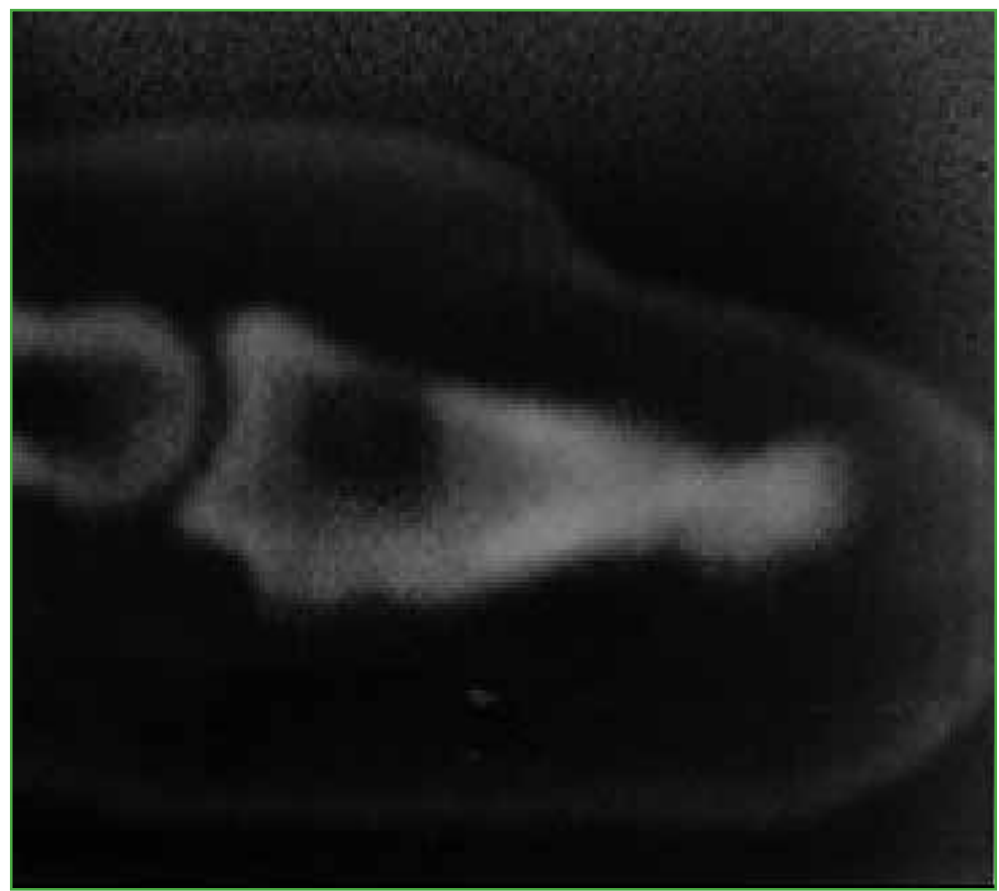

Figura 8. Caso 1. Tomografía computarizada, corte sagital. Imagen osteolítica que compromete la cortical dorsal. Nótese la ausencia de calcificación central y esclerosis reactiva. 

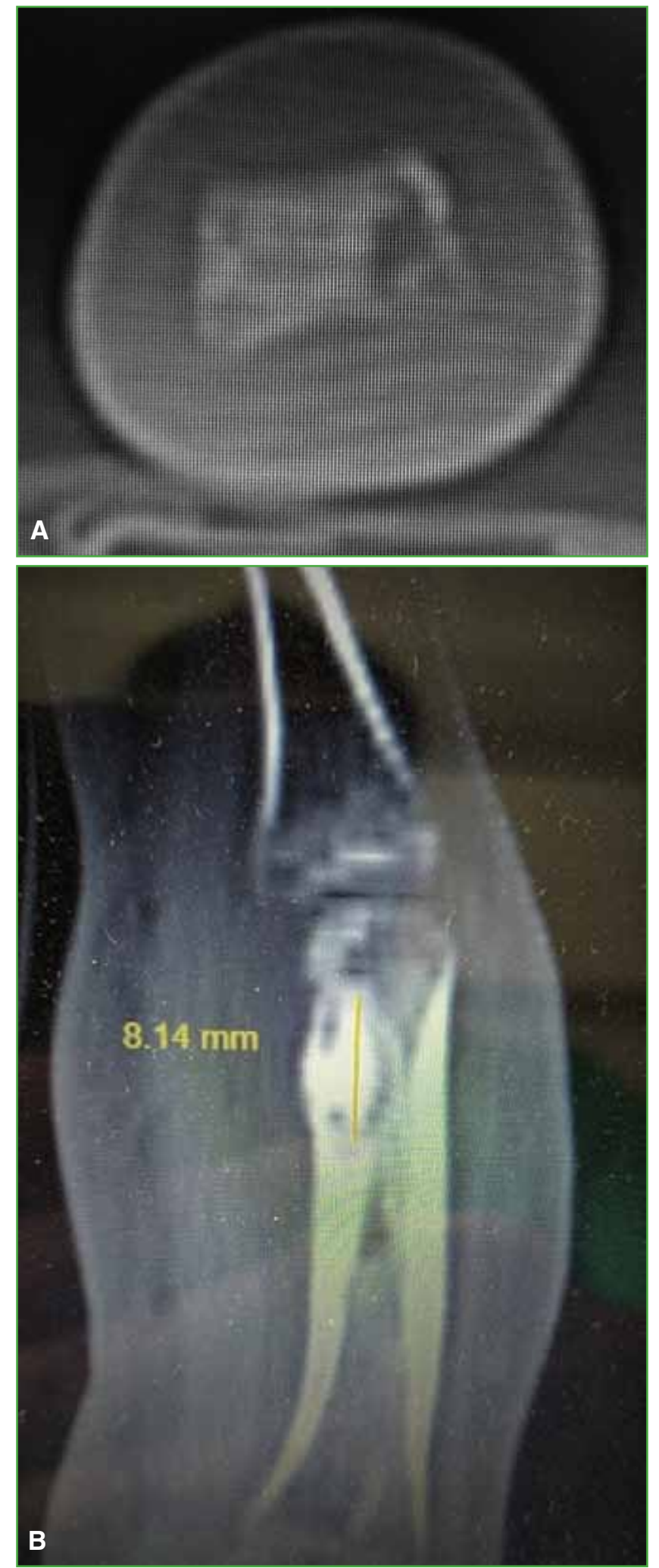

Figura 9. A. Caso 2. Tomografía computarizada. Nótese la imagen osteolítica con calcificación central correspondiente al nido en el cóndilo de la segunda falange. B. Caso 4. Medición del diámetro máximo de la lesión. 
A partir del análisis bibliográfico y de nuestra experiencia limitada, proponemos, ante la sospecha diagnóstica, solicitar, en primer lugar, radiografías simples y complementarlas luego con la TC; en la mayoría de los casos, esto será suficiente para indicar el tratamiento. Cuando el nido no esté mineralizado (como en el caso 1), las radiografías y la TC, en ocasiones, son insuficientes para reforzar el diagnóstico presuntivo, y se puede recurrir tanto a la RM como a la gammagrafía.

Consideramos importante tener en cuenta que la RM, con cierta frecuencia, "magnifica" la lesión, es habitual observar un edema óseo en toda la falange afectada y en las partes blandas adyacentes, esto puede confundir al médico y orientarlo a sospechar un cuadro infeccioso o una neoplasia maligna. Tanto el caso 1 como el caso 4 fueron derivados de otros centros con sospecha de neoplasia maligna sobre la base de las radiografías que mostraban imágenes osteolíticas y la RM en la cual no se lograba visualizar el nido, con un gran edema óseo y de partes blandas (Figura 10).
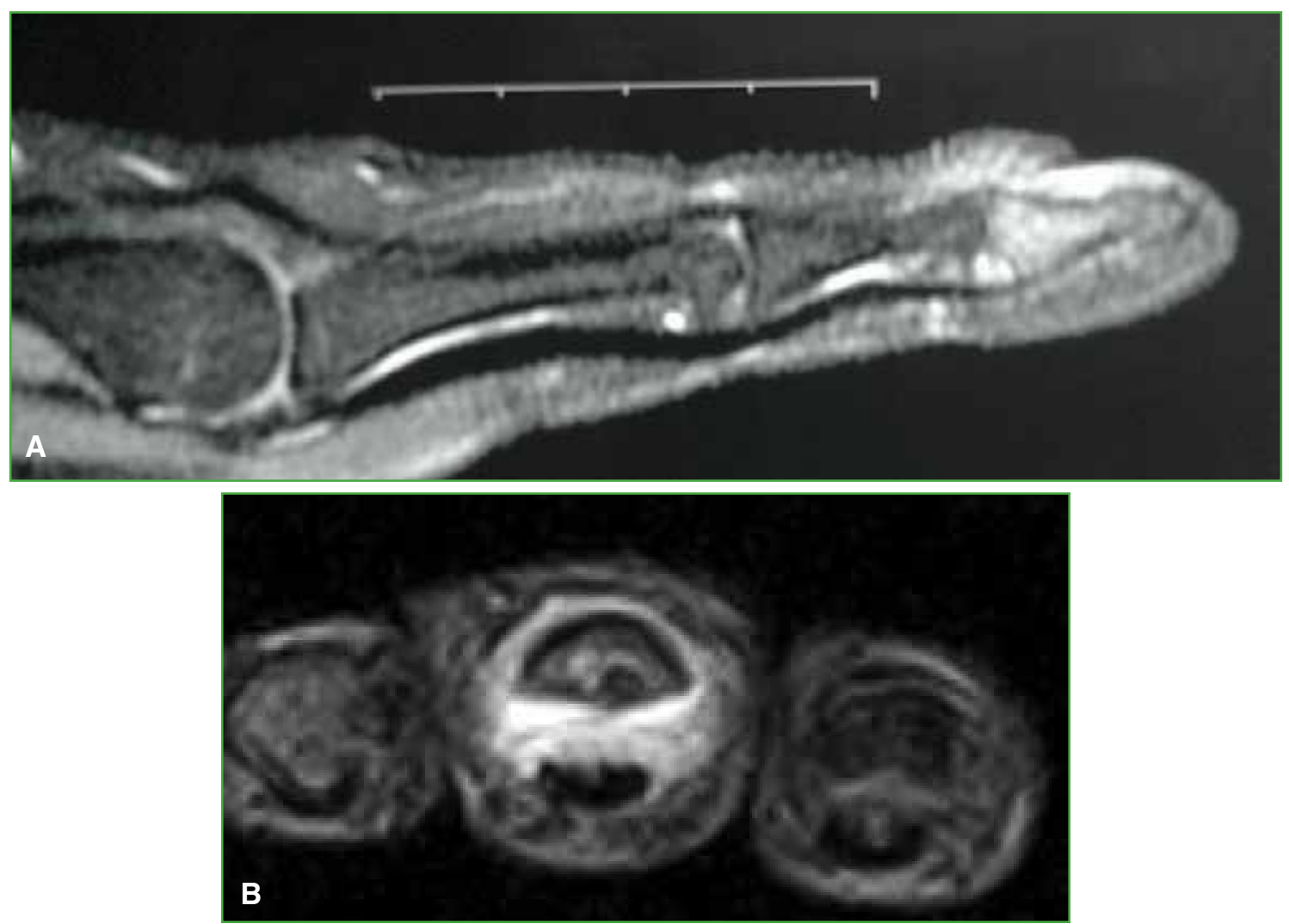

Figura 10. A. Caso 1. Resonancia magnética, corte sagital. Imagen hiperintensa (T2) con edema óseo en la falange distal y de partes blandas. B. Caso 4. Resonancia magnética, corte axial. Obsérvese el gran edema de partes blandas. El nido puede visualizarse en la cortical volar.

Ante un paciente con dolor digital subagudo o crónico, sin un traumatismo, las siguientes manifestaciones clínicas deben alertar al médico sobre un OO:

- dolor nocturno o continuo que cede con antinflamatorios no esteroides, ${ }^{6,8,11,27,28}$

- dolor palpatorio, ${ }^{18}$

- aumento del volumen digital/tumefacción, ${ }^{8,11,15,16,18}$

- aumento del tamaño de la lámina ungueal (compromiso de la falange distal), ${ }^{8,11,15-18}$

- sinovitis (afección epifisaria), ${ }^{7,11}$

- hiperhidrosis..$^{8,15,18}$ 


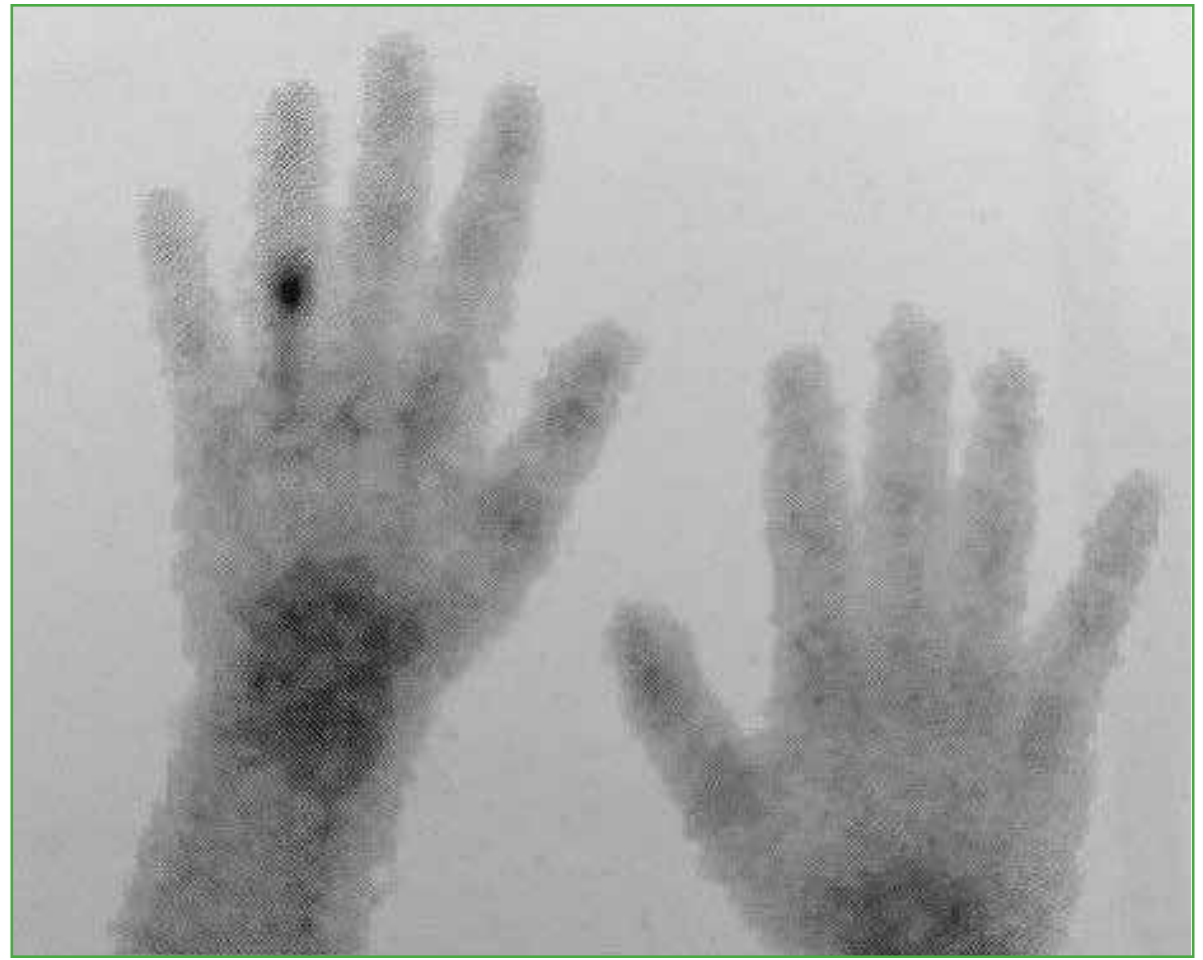

Figura 11. Caso 4. Centellograma óseo en tres fases. Se observa el aumento de la captación en la falange proximal.

El diagnóstico diferencial es clínico y por imágenes, y se debe establecer entre las siguientes entidades:

- tumor glómico, ${ }^{16,18,21}$

- osteomielitis, ${ }^{7,9,11,14,16,18}$

- tenosinovitis,, 11

- quiste epidermoide de inclusión, $, 114,16,18$

- periostitis reactiva, ${ }^{9}$

- fístula arteriovenosa, ${ }^{14,16}$

- neoplasia. ${ }^{7,14,23,29}$

En concordancia con otras publicaciones, ${ }^{3-5,5,11,18,20,22,23,30}$ los pacientes aquí presentados habían realizado múltiples consultas previas, muchas veces, fueron diagnosticados erróneamente y recibieron tratamientos innecesarios que, a menudo, retrasan el diagnóstico.

El tratamiento clásico del $\mathrm{OO}$ consiste en la resección en bloque o el curetaje de la lesión, con injerto óseo o sin él. En los últimos años, ha ganado popularidad el tratamiento percutáneo guiado por TC. Tanto la ablación por radiofrecuencia como la fotocoagulación láser son, en la actualidad, dos de los tratamientos de primera elección en centros de referencia, con tasas de éxito $>90 \% .^{31,32}$ Sin embargo, el empleo de estas técnicas en OO de falanges es escaso y limitado a experiencias aisladas. ${ }^{33}$ Publicaciones recientes ${ }^{34,35}$ advierten sobre la posibilidad de dolor crónico y tumefacción persistente cuando se realiza en las falanges, el dolor se puede atribuir a la lesión térmica del paquete vasculonervioso digital dada su cercanía con la zona por tratar.

La resección de la lesión es el tratamiento definitivo. La recidiva no es frecuente y, si ocurre, debe sospecharse que la resección fue incompleta. Si está localizado en la esponjosa, el curetaje de la lesión suele ser suficiente, mientras que, en los casos de compromiso cortical, la tasa de recidiva es más alta, como sucedió con el paciente 4. Por este motivo, sugerimos la resección en bloque de la cortical afectada. Una alternativa a la resección en bloque es la resección simple de la lesión ampliando los márgenes mediante una fresa de alta velocidad para disminuir la posibilidad de resección incompleta, pues esta es la causa de fracaso más frecuente. 
Cuando hay compromiso de la falange distal, luego de la resección del OO, la tumefacción y el dolor mejoran significativamente, pero persiste el aumento de tamaño de la lámina y el lecho ungueal (Figura 12). ${ }^{16,36}$

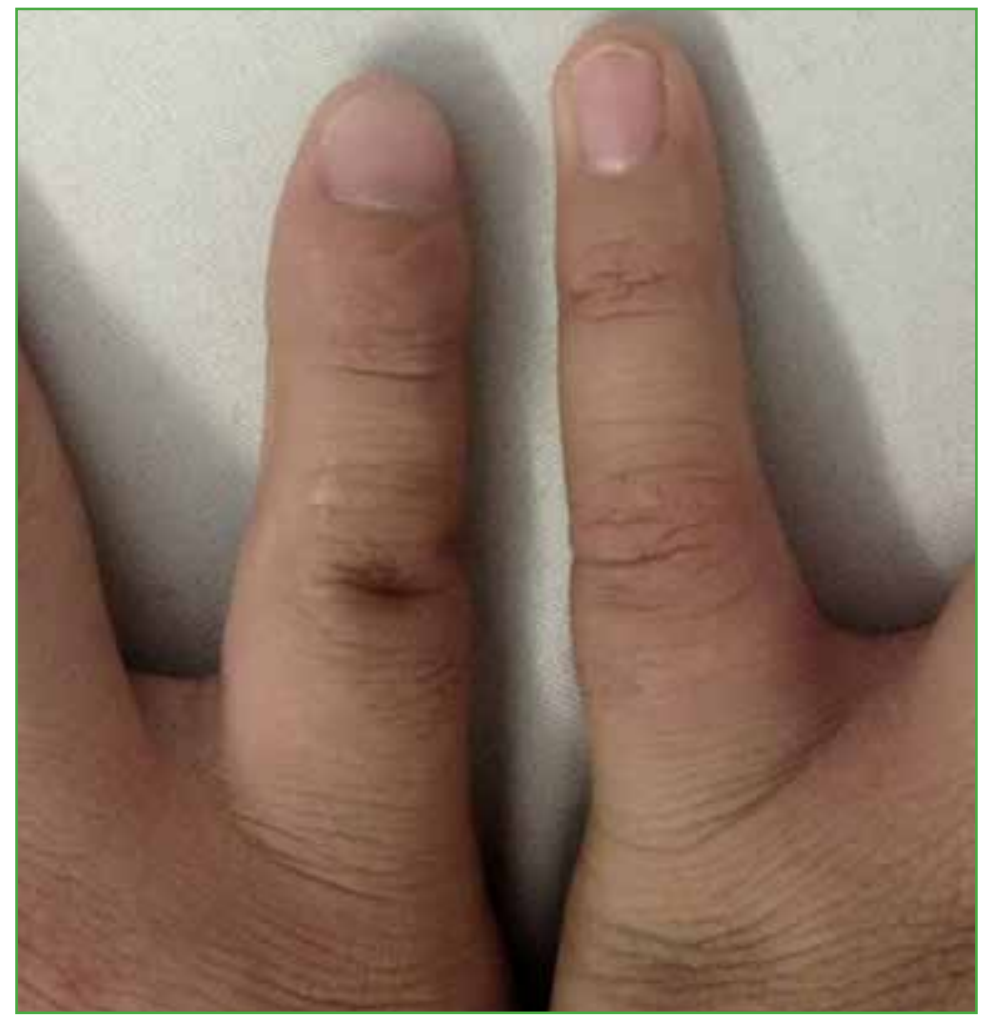

Figura 12. Caso 1. Nótense el aumento del diámetro digital y la uña en aspecto de vidrio de reloj.

\section{CONCLUSIONES}

El OO de falange es un cuadro infrecuente, con manifestaciones clínicas y radiológicas que suelen diferir de las de otras localizaciones. El OO debe pensarse como diagnóstico diferencial ante un paciente que consulta por dolor digital subagudo o crónico que cede con antinflamatorios no esteroides. El aumento del diámetro digital o de la lámina ungueal, el dolor a la palpación, la hiperhidrosis y la sinovitis son manifestaciones relativamente frecuentes y refuerzan la sospecha diagnóstica. La radiografía simple y la TC son los estudios de elección en primera instancia. El tratamiento quirúrgico ya sea mediante el curetaje simple o la resección en bloque obtiene resultados clínicos y radiográficos satisfactorios. Sin embargo, debido a su baja prevalencia, suele pasarse por alto y, de esta manera, se retrasan el diagnóstico y el tratamiento.

Conflicto de intereses: Los autores no declaran conflictos de intereses. 


\section{BIBLIOGRAFÍA}

1. Jaffe HL. "Osteoid-osteoma": A benign osteoblastic tumor composed of osteoid and atypical bone. Arch Surg 1935;31(5):709-28. https://doi.org/10.1001/archsurg.1935.01180170034003

2. Gitelis S, Schajowicz F. Osteoid osteoma and osteoblastoma. Orthop Clin North Am 1989;20(3):313-25. PMID: 2662110

3. Marcuzzi A, Acciaro AL, Landi A. Osteoid osteoma of the hand and wrist. J Hand Surg Br 2002;27(5):440-3. https://doi.org/10.1054/jhsb.2002.0811

4. Ambrosia JM, Wold LE, Amadio PC. Osteoid osteoma of the hand and wrist. J Hand Surg Am 1987;12:794-800. https://doi.org/10.1016/S0363-5023(87)80072-2

5. Burger IM, McCarthy EF. Phalangeal osteoid osteomas in the hand: a diagnostic problem. Clin Orthop Relat Res 2004;(427):198-203. _https://doi.org/0.1097/01.blo.0000142623.97901.39

6. Bednar MS, McCormack RR, Glasser D, Weiland AJ. Osteoid osteoma of the upper extremity. J Hand Surg Am 1993;18(6):1019-25. https://doi.org/10.1016/0363-5023(93)90395-J

7. Georgiev GP, Slavchev SA, Dimitrova IN. Osteoid osteoma of the middle phalanx of the third finger in a child mimicking a malignancy. Folia Medica 2018;60(2):314-17. https://doi.org/10.1515/folmed-2017-0085

8. Giannikas A, Papachristou G, Tiniakos G, Chrysafidis G, Hartofilakidis-Garofalidis G. Osteoid osteoma of the terminal phalanges. Hand 1977;9(3):295-300. https://doi.org/10.1016/S0072-968X(77)80120-4

9. Forriol Brocal F, Martin V, Grasa Muro JL. Osteoma osteoide en falange de la mano. Rev Esp Cir Osteoart 1998;33:83-86. http://hdl.handle.net/10550/39954

10. Soler JM, Pizà G, Aliaga F. Special characteristics of osteoid osteoma in the proximal phalanx. J Hand Surg Br 1997;22:793-7. https://doi.org/10.1016/S0266-7681(97)80451-2

11. Andalib A, Sajadie-Khajouei S. Osteoid osteoma of distal phalanx: A rare disorder and review of literature. $J$ Res Med Sci 2013;18(3):264-6. PMID: 23930128

12. Nakatsuchi Y, Sugimoto Y, Nakano M. Osteoid osteoma of the terminal phalanx. J Hand Surg Br 1984;9(2):201-3. https://doi.org/10.1016/S0266-7681(84)80032-7

13. Esquerdo J, Fernandez CF, Gomar F. Pain in osteoid osteoma: histological facts. Acta Orthop Scand 1976;47(5):520-4. https://doi.org/10.3109/17453677608988731

14. Foucher G, Lemarechal P, Citron N, Merle M. Osteoid osteoma of the distal phalanx: a report of four cases and review of the literature. J Hand Surg Br 1987;12(3):382-6. https://doi.org/10.1016/0266-7681_87_90194-X

15. Rosborough D. Osteoid osteoma: report of a lesion in the terminal phalanx of a finger. J Bone Joint Surg Br 1966;48(3):485-7. PMID: 5913139

16. Bowen C, Dzus A, Hardy D. Osteoid osteomata of the distal phalanx. J Hand Surg Br 1987;12.3:387-90. https://doi.org/10.1016/0266-7681_87_90195-1

17. Levy Y, Rosenheck S, Greiff G. Osteoid osteoma of the distal phalanx of the thumb. Acta Orthop Scand 1979;50(6):667-9. https://doi.org/10.3109/17453677908991290

18. Meng Q, Watt I. Phalangeal osteoid osteoma. Br J Radiol1989;62(736):321-5. https://doi.org/10.1259/0007-1285-62-736-321

19. Edeiken J, De Palma A, Hodes P. Osteoid osteoma. (Roentgenographic emphasis). Clin Orthop Relat Res 1966;49:201-6. PMID: 5962620

20. Barbari K, Prutki M, Starevi D, Seiwerths S, Bojani I. Rare localization of osteoid osteoma - Distal phalanx of the ring finger. Acta Med Croatica 2016;70(3):191-5. https://hrcak.srce.hr/174749

21. Assoun J, Richardi G, Railhac J, Baunin C, Fajadet P, Giron J, et al. Osteoid osteoma: MR imaging versus CT. Radiology 1994;191(1):217-23. https://doi.org/10.1148/radiology.191.1.8134575

22. Zampa V, Bargellini I, Ortori S, Faggioni L, Cioni R, Bartolozziet C. Osteoid osteoma in atypical locations: the added value of dynamic gadolinium-enhanced. MR imaging. Eur J Radiol 2009;71(3):527-35. https://doi.org/10.1016/j.ejrad.2008.05.010

23. Melamud K, Drapé, JL, Hayashi D, Roemer F, Zentner J, Guermazi A. Diagnostic imaging of benign and malignant osseous tumors of the fingers. Radiographics 2014;34(7):1954-67. https://doi.org/10.1148/rg.347130031

24. Spouge A, Thain L. Osteoid osteoma: MR imaging revisited. Clin Imaging 2000;24(1):19-27. https://doi.org/10.1016/S0899-7071(00)00157-1

25. Matiets M. Osteoid osteoma. The role of bone scans in diagnosis and surgery. J Nucl Med Technol 1986;14(3):138-42. http://tech.snmjournals.org/content/14/3/138.abstract 
26. Helms C. Osteoid osteoma. The double density sign. Clin Orthop Relat Res 1987;(222):167-73. PMID: 3621716

27. Doyle L, Ruby L, Nalebuff E, Belsky M. Osteoid osteoma of the hand. J Hand Surg Am1985;10:408-10. https://doi.org/10.1016/S0363-5023(85)80045-9

28. Crosby LA, Murphy RP. Subperiosteal osteoid osteoma of the distal phalanx of the thumb. J Hand Surg Am 1988;13(6):923-25. https://doi.org/10.1016/0363-5023(88)90272-9

29. Mirra J, Kameda N, Rosen G, Eckardt J. Primary osteosarcoma of toe phalanx: first documented case. Review of osteosarcoma of short tubular bones. Am J Surg Pathol 1988;12(4):300-7. https://doi.org/10.1097/00000478-198804000-00007.

30. Brown R, Russell J, Zook E. Osteoid osteoma of the distal phalanx of the finger: a diagnostic challenge. Plast Reconstr Surg 1992;90(6):1016-21. PMID: 1448496

31. Rosenthal D, Hornicek F, Wolfe M, Jennings L, Gebhardt M, Mankin H. Percutaneous radiofrequency coagulation of osteoid osteoma compared with operative treatment. J Bone Joint Surg Am 1988;80:815-21. https://doi.org/10.2106/00004623-199806000-00005

32. Sans N, Galy-Fourcade D, Assoun J, Jarlaud T, Chiavassa H, Bonnevialle P, et al. Osteoid osteoma: CT-guided percutaneous resection and follow-up in 38 patients. Radiology 1999;212:687-92. https://doi.org/10.1148/radiology.212.3.r99se06687

33. Zouari L, Bousson V, Hamzé, Roulot E, Roqueplan F, Laredo J. CT-guided percutaneous laser photocoagulation of osteoid osteomas of the hands and feet. Eur Radiol 2008;18(11):2635-41. https://doi.org/10.1007/s00330-008-1045-3

34. Harrod CC, Boykin RE, Jupiter JB. Pain and swelling after radiofrequency treatment of proximal phalanx osteoid osteoma: case report. J Hand Surg Am 2010;35(6):990-4. https://doi.org/10.1016/j.jhsa.2010.03.012

35. Ramos L, Santos JA, Santos G, Guiral J. Radiofrequency ablation in osteoid osteoma of the finger. J Hand Surg Am 2005;30(4):798-802. https://doi.org/10.1016/j.jhsa.2005.03.009

36. Sullivan M. Osteoid osteoma of the fingers. Hand 1971;3(2):175-8. https://doi.org/10.1016/0072-968X(71)90040-4 